TRANSACTIONS OF THE

AMERICAN MATHEMATICAL SOCIETY

Volume 354, Number 4, Pages 1421-1434

S 0002-9947(01)02950-6

Article electronically published on December 5, 2001

\title{
GLOBAL EXISTENCE FOR A QUASI-LINEAR EVOLUTION EQUATION WITH A NON-CONVEX ENERGY
}

\author{
EDUARD FEIREISL AND HANA PETZELTOVÁ
}

\begin{abstract}
We establish the existence of global in time weak solutions to the initial-boundary value problem related to the dynamics of coherent solid-solid phase transitions in viscoelasticity. The class of the stored energy functionals includes the double well potential, and a general convolution damping term is considered.
\end{abstract}

\section{InTRODUCTION}

We establish the existence of global in time weak solutions to the initial-boundary value problem related to the dynamics of coherent solid-solid phase transitions in viscoelasticity. A hyperelastic material is one for which the Piola-Kirchhoff stress tensor is given by $\partial W(\nabla u)$ where $u$ is the displacement vector and $W$ the stored energy function. On the other hand, experience shows that the stress in certain viscoelastic media can depend not only on the present value of strain but on the temporal history of the dynamical process. Under these circumstances, the equations of motion can be written in the form

$$
u_{t t}^{i}-\partial_{x_{j}}\left(\frac{\partial W}{\partial F_{i, j}}(\nabla u)+k *\left[L_{i, j} \nabla u\right]_{t}\right)+\frac{\partial G}{\partial u^{i}}(u)=0, \quad i=1, \ldots, M,
$$

where

$$
\begin{gathered}
u: \Omega \subset R^{N} \mapsto R^{M}, \nabla u=\left\{\frac{\partial u^{i}}{\partial x_{j}}\right\}, \quad i=1, \ldots, M, j=1, \ldots, N, \\
L_{i, j} F=L_{i, j}^{k, l} F_{k, l} \text { for any matrix } F \in R^{M \times N}, \\
G: R^{M} \mapsto R \text { is a continuously differentiable potential, }
\end{gathered}
$$

and the symbol $*$ stands for the time convolution

$$
k * v(t)=\int_{0}^{t} k(t-s) v(s) \mathrm{d} s .
$$

Repeated indices are always summed, regardless of their position.

We shall suppose the medium occupies a bounded domain $\Omega$ with fixed regular boundary on which the function $u$ satisfies

$$
\left.u\right|_{\partial \Omega}=0 .
$$

Received by the editors February 19, 2000 and, in revised form, August 29, 2001.

2000 Mathematics Subject Classification. Primary 35Q72, 74D10, 45K05.

Work supported by Grant A1019002 GA AVCR. 
The original state of the body is determined by the initial conditions

$$
u(0)=u_{0}, u_{t}(0)=u_{1} .
$$

Ball et al. [1 proposed a one dimensional analogue of (1.1) with the convolution term replaced by the instantaneous damping $-u_{x x t}$ as a simple model that should display the dynamical development of fine structure observed in various crystalline solids. The stored energy function $W$ is typically a non-convex double well potential but we consider a more general situation:

$$
W=W(F): R^{M \times N} \mapsto[0, \infty), W \text { twice continuously differentiable on } R^{M \times N} ;
$$

$$
\begin{gathered}
\sup _{|Z| \leq 1} W(F+Z) \leq c\left(1+W(F)+|F|^{2}\right) \text { for all } F \in R^{M \times N} ; \\
V(F) \equiv W(F)+\Lambda|F|^{2} \text { is convex for a certain } \Lambda \geq 0 .
\end{gathered}
$$

Note that the above hypotheses allow for the power-like stress-strain relation given by the stored energy function

$$
\begin{gathered}
W(F)=\sum_{i=1}^{n} a_{i}\|E\|^{q_{i}}+b_{i}(\operatorname{tr}(E))^{p_{i}}, \\
2 \leq q_{1}<q_{2}<\cdots<q_{n}, 2 \leq p_{1}<p_{2}<\cdots<p_{n}, a_{i}, b_{i} \in R, a_{n}, b_{n}>0,
\end{gathered}
$$

where $E$ stands for the (linearized) strain tensor

$$
E=(1 / 2)\left(F+F^{T}\right)=(1 / 2)\left(\nabla u+(\nabla u)^{T}\right), \quad M=N .
$$

The condition (1.6) ensures "boundedness from below" of the second differential $\partial^{2} W$ as required by the technique of the existence proof. The constitutive law (1.7) includes, of course, some physically relevant materials, in particular, the St. Venant-Kirchhoff material. On the other hand, most of the polyconvex stored energy functions arising in hyperelasticity like the Ogden material are not compatible with our hypotheses because they must be singular for det $F \rightarrow 0$. Also their second differential is usually not bounded from below as required by (1.6) (see e.g. Ciarlet [2] Chapters 3,4]).

The problem with instantaneous damping can be viewed as a particular case of (1.1) where the kernel $k$ is taken the Dirac mass at zero and $L_{i, j}^{k, l}=\delta_{i, k} \delta_{j, l}$. What makes this problem interesting is that it possesses a Lyapunov function - the free energy

$$
E\left[u, u_{t}\right]=\int_{\Omega} \frac{1}{2}\left|u_{t}\right|^{2}+W(\nabla u)+G(u) \mathrm{d} x
$$

which favours formation of finer and finer phase mixtures. These are minimizing sequences converging weakly but not strongly to zero in the Sobolev space $W_{0}^{1,2}(\Omega) \times L^{2}(\Omega)$. The existence of globally defined solutions to this problem was established by Pego [13] in the case $M=N=1$ and Rybka [16] in the multidimensional setting. Both results make use of the transformation introduced in [13] and the technical difficulties of this approach result in rather severe hypotheses imposed on the free energy potential $W$ in [16. More recently, Friesecke and Dolzmann [7] used a completely different technique based on implicit time discretization to generalize the existence result of Rybka [16] to a rather general class of potentials which, however, does not include the physically relevant case (1.7). They also allow for a general viscosity term $-\operatorname{div}\left(L_{i, j} \nabla u_{t}\right)$. 
From the possible applications point of view, however, the model with instantaneous damping exhibits a striking difference between statics and dynamics conjectured by Ball et al. [1] and confirmed by Friesecke and McLeod [8]: While the static picture predicts infinitely many fine patterns minimizing the energy, the solutions of the evolution equation develop patterns of possibly small but still finite length scale. Moreover, the instantaneous damping prevents propagation of singularities (see Rybka [17]) which puts yet another question of applicability of this model to the dynamic formation of microstructures.

Probably the most natural explanation why the dynamic fails to realize the global minimizing sequences is the parabolic character of (1.1) with instantaneous damping. From the mathematical point of view, it is obvious one has to introduce a dissipative term to make the problem tractable by existing analytical methods. However, the dissipation mechanism induced by the instantaneous damping seems unnecessarily strong. For instance, the static picture of transition layers (cf. 8], 17]) seems inherent to parabolic problems with dominating diffusion terms not shared by the problems with regularizing interfacial energy as the Allen-Cahn or Cahn-Hilliard equations.

The presence of a genuine convolution type damping term, i.e., the kernel $k$ is a nonincreasing locally integrable function on $[0, \infty)$, renders the equation (1.1) a problem of different character. For simplicity, we restrict ourselves to the case

$$
k(t)=\frac{t^{\alpha-1}}{\Gamma(\alpha)}, \quad 0<\alpha<1 \text { for } t>0,
$$

related to the problems with fractional time derivatives discussed by Fašangová and Prüss [6], Clément et al. 3] and others. Indeed taking the Fourier transform of $k$ (we tacitly assume $k \equiv 0$ on $(-\infty, 0)$ ), we have

$$
\mathcal{F}[k](\xi)=(\mathrm{i} \xi)^{-\alpha}
$$

which gives some sense to the commonly used notation $k * v_{t}=\partial_{t}^{1-\alpha} v$. Accordingly, the resulting problem is a mathematical idealization of the intermediate level between parabolic and hyperbolic equations close to the former for $\alpha \rightarrow 0$ and resembling the latter when $\alpha \rightarrow 1$.

Taking the scalar product of (1.1) with $u_{t}$ and integrating by parts produces (formally) the energy equality

$$
\frac{d}{d t} E(t)+\int_{\Omega} L_{i, j}^{k, l} \partial_{x_{j}}\left[k * u_{t}^{i}\right] \partial_{x_{l}} u_{t}^{k} \mathrm{~d} x=0 .
$$

Now, we want the bilinear form

$$
\langle\langle v, w\rangle\rangle=\int_{\Omega} L_{i, j}^{k, l}\left(\partial_{x_{j}} v^{i}\right)\left(\partial_{x_{l}} w^{k}\right) \mathrm{d} x
$$

to be a scalar product on the space $W_{0}^{1,2}(\Omega)$ (as a matter of fact, one should write $\left[W_{0}^{1,2}(\Omega)\right]^{M}$ but we shall make no distinction between the scalar and vector function space notation in what follows). To this end, we shall assume the ellipticity condition

$$
L_{i, j}^{k, l}=L_{k, l}^{i, j} \text { and } L_{i, j}^{k, l} \eta_{i} \eta_{k} \xi_{j} \xi_{l} \geq \lambda|\xi|^{2}|\eta|^{2}, \lambda>0, \quad \text { for all } \xi \in R^{N}, \eta \in R^{M} .
$$


Typically, one takes

$$
L_{i, j} \nabla u=(1 / 2)\left(\nabla u+(\nabla u)^{T}\right),
$$

i.e., the symmetric part of the linearized strain.

Due to the presence of the memory term, the equation does not generate a semiflow in the natural energy space and the energy is in general not decreasing along trajectories. However, one can integrate (1.9) with respect to time to produce the term

$$
\int_{0}^{\tau}\left\langle\left\langle k * u_{t}, u_{t}\right\rangle\right\rangle \mathrm{d} t
$$

which is dissipative. More precisely, the kernel $k$ happens to be of positive type (see e.g. Gripenberg et al. [10]) and one has

$$
\int_{0}^{\tau}\langle\langle[k * v], v\rangle\rangle \mathrm{d} t \geq \int_{0}^{\tau}\langle\langle a * v, a * v\rangle\rangle \mathrm{d} t
$$

where the kernel $a$ is given by the formula

$$
a(t)=t^{\frac{\alpha}{2}-1} \sqrt{\cos \left(\frac{\alpha \pi}{2}\right)} / \Gamma\left(\frac{\alpha}{2}\right) .
$$

(see [10, Theorem 16.5.1]).

The problem (1.1) - (1.3) was studied by Engler [5] and Petzeltová and Prüss 14], the space dimension $N$ and the number of equations $M$ being reduced to one in both cases. They obtained strong and globally defined solutions provided the growth rate of the stored energy function $W$ and the order $1-\alpha$ of the fractional derivative appearing in the damping term were suitably related. In particular, the problem in one space dimension possesses a globally defined solution for any $W(F) \approx|F|^{p}$ provided $\alpha<1 / 3$ (cf. [5, 14]). As we will show in Theorem 1.1 below, this hypothesis is far from being optimal even in several space dimensions provided $N \leq 3$.

In contrast to the scalar case posed on an open spatial interval, the problem in several space dimensions has as yet largely withstood a successful mathematical treatment though some related results for linear equations were obtained by Engler [5].

In order to formulate our main result, we need some growth conditions imposed on the lower order terms represented by the potential $G$. Being aware that the only application we have in mind is $G(u)=1 / 2|u|^{2}$ (cf. Ball et al. [1]), we suppose

$$
G \in C^{2}\left(R^{M}\right), G(u) \geq 0,\left|\frac{\partial^{2} G}{\partial u^{2}}\right| \leq c \text { for all } u \in R^{M} .
$$

Having collected all the necessary hypotheses we can now give a precise statement of the existence theorem proved in this paper.

Theorem 1.1. Let $\Omega \subset R^{N}$ be a bounded regular domain. Assume the stored energy function $W$ satisfies the hypotheses (1.4) - (1.6), the kernel $k$ is given by (1.8), the ellipticity condition (1.11) holds for $L_{i, j}$, and $G$ is as in (1.14).

Finally, we suppose

$$
0<\alpha<\min \left\{1 ; \frac{4}{2+N}\right\} .
$$

Then given a time $T>0$ and the initial data

$$
u_{0} \in W^{1, \infty}(\Omega),\left.u_{0}\right|_{\partial \Omega}=0, u_{1} \in L^{2}(\Omega)
$$


the problem (1.1)-(1.3) possesses a weak solution $u$ on the set $(0, T) \times \Omega$. Specifically, we have

$$
\begin{gathered}
u \in C^{\beta}\left([0, T] ; W_{0}^{1,2}(\Omega)\right), 0<\beta<(1-\alpha) / 2, u_{t} \in C\left([0, T] ; L_{\text {weak }}^{2}(\Omega)\right), \\
E\left[u, u_{t}\right] \in L^{\infty}(0, T), k * u_{t} \in L^{2}\left(0, T ; W_{0}^{1,2}(\Omega)\right), \frac{\partial W}{\partial F}(\nabla u) \in L^{1}\left(0, T ; L^{1}(\Omega)\right),
\end{gathered}
$$

and the equation (1.1) holds in $\mathcal{D}^{\prime}((0, T) \times \Omega)$.

Moreover, the quantity $a * u_{t}$, where the kernel $a$ is given by (1.13), belongs to $L^{2}\left(0, T ; W_{0}^{1,2}(\Omega)\right)$, and the energy inequality

$$
E\left[u, u_{t}\right](\tau)+\int_{0}^{\tau}\left\langle\left\langle a * u_{t}, a * u_{t}\right\rangle\right\rangle \mathrm{d} t \leq E\left[u_{0}, u_{1}\right]
$$

holds for a.a. $\tau \in(0, T)$.

Outside the inevitable energy estimates (see Section 2), our approach hinges on two crucial properties of any family $\left\{u^{n}\right\}$ of solutions of the problem: Compactness of the time derivative $u_{t}^{n}$ in $L^{2}\left(0, T ; L^{2}(\Omega)\right.$ ) (cf. Section 3), and the time propagation (or rather non-propagation) of singularities in the strain tensor $\nabla u^{n}$ (Section 5). Note that the latter property was observed for the problem with instantaneous damping by Friesecke and Dolzmann [7]. However, the technique of the implicit time discretization used in [7] is hard to apply in our setting due to the presence of the memory term. Instead, we shall use the approach based on careful analysis of the defect measure

$$
\omega(t)=\lim _{n \rightarrow \infty} \int_{\Omega}\left(\left|\nabla u^{n}(t)\right|^{2}-|\nabla u(t)|^{2}\right) \mathrm{d} x
$$

where $\nabla u$ is a (weak) limit of $\nabla u^{n}$.

To show compactness of the sequence $\left\{u_{t}^{n}\right\}$, we use a modification of the LionsAubin lemma (see Proposition 3.1) which may be of independent interest.

To conclude this introduction, let us remark that our approach applies directly to the problem with instantaneous damping ( $k$ - the Dirac mass at zero), and, consequently, it generalizes and considerably simplifies the proof of the existence result in [7]. In particular, the hypothesis (1.5) is not necessary in this case.

\section{ENERGy ESTimates}

We derive a priori estimates for any sequence $\left\{u^{n}\right\}$ of (classical) solutions of the problem (1.1)-(1.3) on the time interval $(0, \infty)$ based on the energy equality (1.9). From the hypotheses (1.4), (1.12), (1.14) it follows that

$$
\begin{gathered}
u_{t}^{n} \text { are bounded in } L^{\infty}\left(0, \infty ; L^{2}(\Omega)\right), \\
W\left(\nabla u^{n}\right) \text { are bounded in } L^{\infty}\left(0, \infty ; L^{1}(\Omega)\right),
\end{gathered}
$$

and

$$
a * u_{t}^{n} \text { are bounded in } L^{2}\left(0, \infty ; W_{0}^{1,2}(\Omega)\right)
$$

in terms of the initial energy $E\left[u^{n}(0), u_{t}^{n}(0)\right]$. In particular, we have

$$
u_{t}^{n} \text { bounded in } W^{-\frac{\alpha}{2}, 2}\left(R ; W_{0}^{1,2}(\Omega)\right)
$$


provided we set $u_{t}^{n} \equiv 0$ for negative values of $t$. Here, the spaces $W^{\beta, 2}(I ; Y), \beta \in R$, $Y$ a Hilbert space, are defined as the quotient spaces of restrictions of distributions from

$$
W^{\beta, 2}(R ; Y)=\left\{v \in \mathcal{S}^{\prime}(R, Y) \mid\left(1+|\xi|^{2}\right)^{\beta / 2} \mathcal{F}[v](\xi) \in L^{2}(R, Y)\right\}
$$

to a time interval $I$ (cf. e.g. Triebel [18, Chapters 2.3.3, 4.2.1]).

Now, we can conclude from (2.4) that

$$
u^{n} \text { is bounded in } C^{\beta}\left([0, T] ; W_{0}^{1,2}(\Omega)\right), \quad 0<\beta<(1-\alpha) / 2,
$$

provided $u^{n}(0)$ is bounded in $W_{0}^{1,2}(\Omega)$. Indeed this is a consequence of

Lemma 2.1. Let $X, Y$ be two Hilbert spaces such that

$$
X \hookrightarrow Y
$$

with continuous and dense embeddings. Let $v \in L^{\infty}(0, T ; X)$ such that

$$
v_{t} \in L^{2}(0, T ; Y) \cap W^{-\frac{\alpha}{2}, 2}(0, T ; X), \quad 0<\alpha<1 .
$$

Then

$$
\|v(t)-v(s)\|_{X} \leq c|t-s|^{\beta}, \quad t, s \in[0, T], 0<\beta<(1-\alpha) / 2 .
$$

Proof. To begin, note that $v \in C\left([0, T] ; X_{\text {weak }}\right)$ so (2.7) makes sense. In accordance with (2.6), there is a function $w \in W^{-\frac{\alpha}{2}, 2}(R ; X)$ such that

$$
\left.w\right|_{(0, T)}=v_{t}
$$

Consequently, we have

$$
\|v(t)-v(s)\|_{X}=\|z(t)-z(s)\|_{X}, \quad t, s \in[0, T],
$$

where

$$
z=\mathcal{F}^{-1}\left(\frac{\mathcal{F}[w](\xi)}{\mathrm{i} \xi}\right)
$$

Thus

$$
\|v(t)-v(s)\|_{X}=\frac{1}{2 \pi}\left\|\int_{R}\left(e^{\mathrm{i} t \xi}-e^{\mathrm{i} s \xi}\right) \frac{\mathcal{F}[w](\xi)}{\mathrm{i} \xi} \mathrm{d} \xi\right\|_{X}
$$

where

Seeing that

$$
\mathcal{F}[w](\xi)=\left(1+|\xi|^{2}\right)^{\frac{\alpha}{4}} G(\xi), \quad G \in L_{\xi}^{2}(R ; X)
$$

$$
\left|e^{\mathrm{i} t \xi}-e^{\mathrm{i} s \xi}\right| \leq 2^{1-\delta}|\xi|^{\delta}|t-s|^{\delta} \text { for any } \delta \in[0,1],
$$

the relation (2.7) follows from (2.8) by direct computation.

From (2.5) and uniform boundedness of the energy we get

$$
V\left(\nabla u^{n}\right) \text { bounded in } L^{\infty}\left(0, T ; L^{1}(\Omega)\right)
$$

where $V$ is the convex function from (1.6).

At this stage we need the following assertion.

Lemma 2.2. Under the hypotheses (1.4)-(1.6), we have

$$
\left|\frac{\partial W}{\partial F}(F)\right| \leq c\left(W(F)+F^{2}+1\right) \text { for all } F \in R^{M \times N} .
$$


Proof. In view of (1.5), we get

$$
\sup _{|Z| \leq 1} V(F+Z) \leq c(V(F)+1) .
$$

As $V$ is convex, one has

$$
\frac{\partial V}{\partial F}(F): Z \leq V(F+Z)-v(F) \leq c(V(F)+1)
$$

provided $|Z| \leq 1$.

Thus

$$
\left|\frac{\partial V}{\partial F}(F)\right| \leq c\left(W(F)+F^{2}+1\right)
$$

and the desired conclusion follows.

As a straightforward consequence of Lemma 2.2 and (2.9), we get

$$
\left|\frac{\partial W}{\partial F}\left(\nabla u^{n}\right)\right| \text { bounded in } L^{\infty}\left(0, T ; L^{1}(\Omega)\right)
$$

The next step is to take the scalar product of (1.1) with $u^{n}$. After a straightforward computation, we obtain

$$
\begin{gathered}
\int_{0}^{T} \int_{\Omega} \frac{\partial W\left(\nabla u^{n}\right)}{\partial F}: \nabla u^{n} \mathrm{~d} x \mathrm{~d} t \\
=\int_{0}^{T} \int_{\Omega}\left|u_{t}^{n}\right|^{2} \mathrm{~d} x \mathrm{~d} t-\int_{\Omega}\left[u_{t}^{n}(t) \cdot u^{n}(t)\right]_{t=0}^{t=T} \mathrm{~d} x-\int_{0}^{T}\left\langle\left\langle k * u_{t}^{n}, u^{n}\right\rangle\right\rangle \mathrm{d} t \\
-\int_{0}^{T} \int_{\Omega} \frac{\partial G}{\partial u}\left(u^{n}\right) \cdot u^{n} \mathrm{~d} x \mathrm{~d} t
\end{gathered}
$$

where the right-hand side is bounded by virtue of the estimates (2.1), (2.3), (2.5). Note, in particular, that (2.3), 2.5) imply

$$
k * u_{t}^{n} \text { bounded in } L^{2}\left(0, T ; W_{0}^{1,2}(\Omega)\right) .
$$

Thus the right-hand side of (2.11) is bounded and, consequently,

$$
\frac{\partial V}{\partial F}\left(\nabla u^{n}\right): \nabla u^{n} \text { are bounded in } L^{1}((0, T) \times \Omega) .
$$

Since we have

$$
\frac{\partial V}{\partial F}\left(\nabla u^{n}\right): \nabla u^{n}=V\left(\nabla u^{n}\right)+V^{*}\left(\frac{\partial V}{\partial F}\left(\nabla u^{n}\right)\right),
$$

where $V^{*}$ is the convex conjugate function, we deduce, by virtue of (2.13),

$$
V^{*}\left(\frac{\partial V}{\partial F}\left(\nabla u^{n}\right)\right) \text { is bounded in } L^{1}((0, T) \times \Omega) .
$$




\section{A COMPACTNESS RESUlT}

The most important property of the energy estimates presented above is that they ensure compactness of the time derivatives $\left\{u_{t}^{n}\right\}$ in the Hilbert space $L^{2}\left((0, T) \times L^{2}\right)$. Such a property for the system with instantaneous damping term follows directly from Lions-Aubin lemma.

Proposition 3.1. Let $X \subset Z$ be two Hilbert spaces,

$$
X \hookrightarrow Z \text { densely and compactly. }
$$

Consider the Hilbert space

$$
\mathcal{X}=\left\{v \mid v \in L^{2}(0, T ; Z) \cap W^{-\frac{\alpha}{2}, 2}(0, T ; X), v_{t} \in L^{2}(0, T ; Z)\right\}
$$

for $0<\alpha<1$.

Then

$$
\mathcal{X} \hookrightarrow L^{2}\left(0, T ;[X, Z]_{\theta}\right) \text { compactly for any } \theta>\frac{\alpha}{\alpha+2}
$$

where $[X, Y]_{\theta}$ is the interpolation space.

Remark. See Lions and Magenes [12, Chapter 1] for the definition of the interpolation space $[X, Z]_{\theta}$.

Proof. We have

$$
\mathcal{X} \hookrightarrow W^{-\frac{\alpha}{2}, 2}(0, T ; X) \text { and } \mathcal{X} \hookrightarrow W^{1,2}(0, T ; Z) .
$$

Thus, by interpolation (cf. Lions and Magenes [12, Chapter 1, Theorem 12.4 and (9.24)]),

$$
\mathcal{X} \hookrightarrow L^{2}\left(0, T ;[X, Z]_{\theta}\right) \text { with } \theta=\frac{\alpha}{\alpha+2} .
$$

As $[X, Z]_{\theta_{1}}$ is compactly embedded to $[X, Z]_{\theta_{2}}$ for $\theta_{2}>\theta_{1}$, the desired conclusion follows from the Lions-Aubin lemma (see Lions [11, Chapter 1, Theorem 5.1]).

Now, we consider the scale of Hilbert spaces $H^{\beta}$ defined as

$$
H^{\beta}=D\left(A^{\beta / 2}\right), \quad \beta \in R,
$$

where

$$
D(A)=\left[W^{2,2} \cap W_{0}^{1,2}(\Omega)\right]^{M},(A v)^{i}=-\Delta v^{i}, \quad i=1, \ldots, M .
$$

It is well-known that

$$
H^{\beta} \subset C_{0}^{1}(\Omega) \text { for } \beta>1+\frac{N}{2} .
$$

Consequently, under the assumptions and notation of Section 2 we have

$$
u_{t t}^{n} \text { bounded in } L^{2}\left(0, T ; H^{-\beta}\right), \quad \beta>1+\frac{N}{2} .
$$

Now, we can apply Proposition 3.1 to the sequence $\left\{u_{t}^{n}\right\}$ with the choice

$$
X=H^{1}=W_{0}^{1,2}(\Omega), Z=H^{-\beta}, \quad \beta>1+\frac{N}{2} .
$$

Seeing that

$$
L^{2}(\Omega)=H^{0}=\left[H^{1}, H^{-\beta}\right]_{\theta_{0}} \text { with } \theta_{0}=\frac{1}{1+\beta}
$$

we can use Proposition 3.1 to conclude

$$
u_{t}^{n} \text { precompact in } L^{2}\left(0, T ; L^{2}(\Omega)\right) \text { provided } 0<\alpha<\min \left\{1 ; \frac{4}{2+N}\right\}
$$

which is precisely the hypothesis (1.15). 


\section{Approximations AND COMPACTNESS}

The a priori estimates derived in Section [2 are perfectly compatible with the Faedo-Galerkin approximation scheme. More specifically, we consider a sequence of finite-dimensional spaces

$$
\begin{aligned}
Y_{n}=\operatorname{span}\{ & {\left[\phi_{1}, \ldots, \phi_{n}\right]^{M} \mid \phi_{i} \text { the eigenfunctions of the operator } } \\
& -\Delta \text { on } \Omega \text { with the zero Dirichlet boundary conditions }\} .
\end{aligned}
$$

Since both $W$ and $G$ are twice continuously differentiable, we can use 9, Chapter 5 , Theorem 2.1] to construct a sequence of approximate solutions $u^{n} \in C^{2}\left([0, T] ; Y_{n}\right)$ satisfying the integral identity

$$
\begin{gathered}
\frac{\mathrm{d}^{2}}{\mathrm{~d} t^{2}} \int_{\Omega} u^{n} \cdot \phi \mathrm{d} x+\int_{\Omega} \frac{\partial W}{\partial F}\left(\nabla u^{n}\right): \nabla \phi+\nabla_{u} G\left(u^{n}\right) \cdot \phi \mathrm{d} x+\left\langle\left\langle k * u_{t}^{n}, \phi\right\rangle\right\rangle \\
=0 \text { for all } \phi \in Y^{n} \\
u^{n}(0)=u_{0}^{n} \in Y_{n}, u_{t}^{n}(0)=u_{1}^{n} \in Y_{n}
\end{gathered}
$$

where, in general, $T=T(n)>0$.

However, rewriting (4.1) as

$$
\begin{aligned}
& {\left[\int_{\Omega} u_{t}^{n} \cdot \varphi \mathrm{d} x\right]_{t=0}^{t=\tau}+\int_{0}^{\tau} \int_{\Omega} \frac{\partial W}{\partial F}\left(\nabla u^{n}\right): \nabla \varphi+\frac{\partial G}{\partial u}\left(u^{n}\right) \cdot \varphi \mathrm{d} x \mathrm{~d} t+\int_{0}^{\tau}\left\langle\left\langle k * u_{t}^{n}, \varphi\right\rangle\right\rangle \mathrm{d} t} \\
& =\int_{0}^{\tau} \int_{\Omega} u_{t}^{n} \cdot \varphi_{t} \mathrm{~d} x \mathrm{~d} t \text { for any } \tau \in[0, T] \text { and any test function } \varphi \in W^{1,2}\left(0, T ; Y_{n}\right)
\end{aligned}
$$
and choosing $\varphi=u_{t}^{n}$, we obtain the energy inequality

$$
E\left[u^{n}, u_{t}^{n}\right](\tau)+\int_{0}^{\tau}\left\langle\left\langle a * u_{t}^{n}, a * u_{t}^{n}\right\rangle\right\rangle \mathrm{d} t \leq E\left[u_{0}^{n}, u_{1}^{n}\right] \text { for any } \tau \geq 0 .
$$

In particular, one has

$$
\left\|u^{n}(t)\right\|_{X} \leq c(n)\left\|u^{n}(t)\right\|_{L^{2}(\Omega)} \leq c t \text { for } t \in[0, T]
$$

and, consequently, one can continue the solution $u^{n}$ up to $T(n)=\infty$.

The functions $u_{0}^{n}, u_{1}^{n}$ are chosen so that

$$
u_{0}^{n} \rightarrow u_{0} \text { in } W_{0}^{1,2}(\Omega), \int_{\Omega} V\left(\nabla u_{0}^{n}\right) \mathrm{d} x \leq c \text { independently of } n, u_{1}^{n} \rightarrow u_{1} \text { in } L^{2}(\Omega) .
$$

Taking $\varphi=u^{n}$ in (4.3) yields (2.11) and, consequently, all the energy estimates derived formally in Section 2 hold for the sequence of the approximate solutions $u^{n}$. Moreover, as (3.1) follows from the special choice of the basis $\left\{\phi_{i}\right\}$ we have (3.2) as well.

Accordingly, passing to the limit for $n \rightarrow \infty$ and considering subsequences if necessary, we have

$$
\begin{gathered}
u^{n} \rightarrow u \text { in } C\left([0, T] ;\left[W_{0}^{1,2}\right]_{\text {weak }}(\Omega)\right), \\
u_{t}^{n} \rightarrow u_{t} \text { in } C\left([0, T] ; L_{\text {weak }}^{2}(\Omega)\right) \text { and in } L^{2}\left(0, T ; L^{2}(\Omega)\right), \\
a * u_{t}^{n} \rightarrow a * u_{t} \text { weakly in } L^{2}\left(0, \infty ; W_{0}^{1,2}(\Omega)\right),
\end{gathered}
$$




$$
\begin{gathered}
k * u_{t}^{n} \rightarrow k * u_{t} \text { weakly in } L^{2}\left(0, T ; W_{0}^{1,2}(\Omega)\right), \\
\frac{\partial W}{\partial F}\left(\nabla u^{n}\right) \rightarrow \overline{\frac{\partial W}{\partial F}(\nabla u)} \text { weakly in } L^{1}\left(0, T ; L^{1}(\Omega)\right)
\end{gathered}
$$

where (4.10) follows from (2.14) and the Dunford-Pettis theorem. Indeed one has

$$
\lim _{|F| \rightarrow \infty} \frac{V^{*}(F)}{|F|}=\infty
$$

and we can use [4, Chapter 8, Theorem 1.3].

Next, we let $n \rightarrow \infty$ in (4.3) to deduce

$$
\begin{aligned}
& {\left[\int_{\Omega} u_{t} \cdot \varphi \mathrm{d} x\right]_{t=0}^{t=\tau}+\int_{0}^{\tau} \int_{\Omega} \frac{\overline{\partial W}}{\partial F}(\nabla u): \nabla \varphi+\frac{\partial G}{\partial u}(u) \cdot \varphi \mathrm{d} x \mathrm{~d} t+\int_{0}^{\tau}\left\langle\left\langle k * u_{t}, \varphi\right\rangle\right\rangle \mathrm{d} t} \\
& =\int_{0}^{\tau} \int_{\Omega} u_{t} \cdot \varphi_{t} \mathrm{~d} x \mathrm{~d} t \text { for any test function } \varphi \in W^{1,2}\left(0, T ; Y_{n}\right), \quad n=1,2, \ldots .
\end{aligned}
$$

It follows by a simple density argument that (4.11) holds for any test function $\varphi$ such that

$$
\varphi \in L^{2}\left(0, T ; H^{\beta}\right), \varphi_{t} \in L^{2}\left(0, T ; L^{2}(\Omega)\right), \quad \beta>1+\frac{N}{2} .
$$

In particular, one has,

$$
\begin{aligned}
\left.u_{t t}^{i}-\partial_{x_{j}} \overline{\left(\frac{\partial W}{\partial F_{i, j}}(\nabla u)\right.}+k *\left[L_{i, j} \nabla u\right]_{t}\right)+\frac{\partial G}{\partial u^{i}}(u)=0, & \\
& i=1, \ldots, M \text { in } \mathcal{D}^{\prime}((0, T) \times \Omega) .
\end{aligned}
$$

Moreover, $u, u_{t}$ satisfy the initial conditions (1.16).

Thus our ultimate goal is to show

$$
\overline{\frac{\partial W}{\partial F}(\nabla u)}=\frac{\partial W}{\partial F}(\nabla u)
$$

which will complete the proof of Theorem 1.1. This will be done in the last section.

\section{Strong CONVERGENCE OF the GRADients}

Our aim is to show strong convergence of the gradients $\nabla u^{n}$ in the sequence of approximate solutions, more specifically,

$$
\nabla u^{n} \rightarrow \nabla u \text { in } L^{2}((0, T) \times \Omega) .
$$

The main idea is similar to that of Friesecke and Dolzmann 7], namely, that oscillations in $\nabla u^{n}$ cannot occur unless they were present in the initial data. Note however that the technique of the implicit time discretization does not seem to be applicable here because of the non-local character of the dissipative term. Our approach is based on the concept of defect measure and it is rather versatile in the sense it can be applied to the problems with instantaneous damping as well. Moreover, it is compatible with any approximation scheme of Faedo-Galerkin type as well as with the methods based on vanishing viscosity or capillarity.

We introduce the defect measure

$$
\omega=\omega(t)=\lim _{n \rightarrow \infty} \int_{\Omega}\left|\nabla u^{n}(t)\right|^{2}-|\nabla u(t)|^{2} \mathrm{~d} x .
$$


By virtue of Lemma 2.1 and (2.5), the functions

$$
t \mapsto \int_{\Omega}\left|\nabla u^{n}(t)\right|^{2}-|\nabla u(t)|^{2} \mathrm{~d} x
$$

are uniformly bounded and uniformly Hölder continuous and so is $\omega$ whose definition is now fully justified in view of the Arzela-Ascoli theorem. Moreover, by virtue of (4.6), $\omega$ is a non-negative function. Clearly, (5.1) will follow provided we show $\omega \equiv 0$.

To this end, we derive an integral inequality based on (4.3) and (4.11). Let us start with an elementary property of the kernel $k$.

Lemma 5.1. Let $X$ be a Hilbert spaces. Assume that

$$
v \in C([0, T] ; X), v_{t} \in W^{-\frac{\alpha}{2}, 2}(0, T ; X)
$$

Then

$$
\begin{aligned}
& \int_{0}^{T}\left\langle\left\langle\left[k * v_{t}\right](t), v(t)\right\rangle\right\rangle \mathrm{d} t \\
& \quad \geq \frac{1}{2} \int_{0}^{T} k(t)\left(\|v(T-t)\|_{X}^{2}+\|v(t)\|_{X}^{2}\right) \mathrm{d} t-\int_{0}^{T} k(t)\langle\langle v(0), v(t)\rangle\rangle \mathrm{d} t
\end{aligned}
$$

where the symbol $\langle\langle\rangle$,$\rangle stands for the scalar product in X$.

Proof. (i) First take $k_{\delta}$ such that

$$
k_{\delta}(t)=\left\{\begin{array}{l}
0 \quad \text { if } t \leq 0 \\
k(t+\delta) \quad \text { for } t \in[0, T] \\
\max \left\{0, k(T+\delta)+k^{\prime}(T+\delta)(t-T)\right\} \quad \text { for } t>T .
\end{array}\right.
$$

A straightforward computation yields the identity (cf. [10, Chapter 18, Lemma 4.1])

$$
\begin{aligned}
& \int_{0}^{T}\left\langle\left\langle\left[k_{\delta} * v_{t}\right](t), v(t)\right\rangle\right\rangle \mathrm{d} t=\frac{1}{2} \int_{0}^{T} k_{\delta}(t)\left(\|v(T-t)\|_{X}^{2}+\|v(t)\|_{X}^{2}\right) \mathrm{d} t \\
& -\int_{0}^{T} k_{\delta}(t)\langle\langle v(0), v(t)\rangle\rangle \mathrm{d} t-\frac{1}{2} \int_{0}^{T} \int_{0}^{t} k_{\delta}^{\prime}(s)\|v(t-s)-v(t)\|_{X}^{2} \mathrm{~d} s \mathrm{~d} t
\end{aligned}
$$

giving (5.2) as $k_{\delta}$ is nonincreasing. Observe that

$$
\left\|k_{\delta} * u_{t}\right\|_{L^{2}(0, T ; X)} \leq\left\|k_{\delta} * w\right\|_{L^{2}(R ; X)}
$$

where $w \in W^{-\frac{\alpha}{2}, 2}(0, T ; X),\left.w\right|_{(0, T)}=u_{t}$, and, by virtue of the Parseval identity and [15, Chapter 1, Theorem 3.10],

$$
\begin{gathered}
\left\|k_{\delta} * w\right\|_{L^{2}(R ; X)}^{2}=\int_{R}\left|\mathcal{F}\left[k_{\delta}\right](\xi)\right|^{2}\|\mathcal{F}[w](\xi)\|_{X}^{2} \mathrm{~d} \xi \\
\leq c \int_{R}\left(1+|\xi|^{2}\right)^{-\frac{\alpha}{2}}\|\mathcal{F}[w](\xi)\|_{X}^{2} \mathrm{~d} \xi
\end{gathered}
$$

(ii) Now, letting $\delta \rightarrow 0$ we deduce from (5.4) that

$$
k_{\delta} * u_{t} \rightarrow k * u_{t} \text { weakly in } L^{2}(0, T ; X)
$$

which enables us to pass to the limit in (5.2) to deduce the desired conclusion. 
Taking $\phi=u^{n}$ in (4.3), (4.11) we arrive at

$$
\begin{aligned}
\int_{0}^{\tau}\left\langle\left\langle k *\left(u_{t}^{n}-u_{t}\right), u^{n}\right\rangle\right\rangle+\int_{0}^{\tau} \int_{\Omega} \frac{\partial W}{\partial F}\left(\nabla u^{n}\right): \nabla u^{n}-\frac{\partial W}{\partial F}(\nabla u): \nabla u^{n} \mathrm{~d} x \mathrm{~d} t \\
=\int_{0}^{\tau} \int_{\Omega}\left(u_{t}^{n}-u_{t}\right) \cdot u_{t}^{n}+\left(\frac{\partial G}{\partial u}(u)-\frac{\partial G}{\partial u}\left(u^{n}\right)\right) \cdot u^{n} \mathrm{~d} x \mathrm{~d} t \\
\quad+\left[\int_{\Omega}\left(u_{t}^{n}-u_{t}\right) \cdot u^{n} \mathrm{~d} x\right]_{t=\tau}^{t=0} .
\end{aligned}
$$

It follows from (4.6), (4.7) that the right-hand side of (5.5) tends to zero for $n \rightarrow \infty$.

On the other hand, we have

$\int_{0}^{\tau}\left\langle\left\langle k *\left(u_{t}^{n}-u_{t}\right), u^{n}\right\rangle\right\rangle \mathrm{d} t=\int_{0}^{\tau}\left\langle\left\langle k *\left(u_{t}^{n}-u_{t}\right), u^{n}-u\right\rangle\right\rangle \mathrm{d} t+\int_{0}^{\tau}\left\langle\left\langle k *\left(u_{t}^{n}-u_{t}\right), u\right\rangle\right\rangle \mathrm{d} t$ where

$$
\int_{0}^{\tau}\left\langle\left\langle k *\left(u_{t}^{n}-u_{t}\right), u\right\rangle\right\rangle \mathrm{d} t \rightarrow 0 \text { for } n \rightarrow \infty
$$

in view of (4.9). Moreover, making use of Lemma 5.1 for $X=W_{0}^{1,2}, Y=L^{2}(\Omega)$ we deduce

$$
\begin{aligned}
\int_{0}^{\tau}\left\langle\left\langle k *\left(u_{t}^{n}-u_{t}\right), u^{n}-u\right\rangle\right\rangle \mathrm{d} t \\
\geq \lambda \int_{0}^{\tau} k(t)\left(\int_{\Omega}\left|\nabla\left(u^{n}-u\right)(\tau-t)\right|^{2}+\left|\nabla\left(u^{n}-u\right)(t)\right|^{2} \mathrm{~d} x\right) \mathrm{d} t \\
\quad-\int_{0}^{\tau} k(t)\left\langle\left\langle\left(u^{n}-u\right)(0),\left(u^{n}-u\right)(t)\right\rangle\right\rangle \mathrm{d} t \text { for a certain } \lambda>0
\end{aligned}
$$

where

$$
\int_{0}^{\tau} k(t)\left(\int_{\Omega}\left|\nabla\left(u^{n}-u\right)(\tau-t)\right|^{2}+\left|\nabla\left(u^{n}-u\right)(t)\right|^{2} \mathrm{~d} x\right) \mathrm{d} t \rightarrow \int_{0}^{\tau} k(t)(\omega(\tau-t)+\omega(t)) \mathrm{d} t
$$

and

$$
\int_{0}^{\tau} k(t)\left\langle\left\langle\left(u^{n}-u\right)(0),\left(u^{n}-u\right)(t)\right\rangle\right\rangle \mathrm{d} t \rightarrow 0
$$

in accordance with (4.5).

Summarizing the previous estimates we conclude

$$
\liminf _{n \rightarrow \infty} \int_{0}^{\tau}\left\langle\left\langle k *\left(u_{t}^{n}-u_{t}\right), u^{n}\right\rangle\right\rangle \geq \lambda \int_{0}^{\tau} k(t)(\omega(\tau-t)+\omega(t)) \mathrm{d} t .
$$

Finally, we compute

$$
\begin{aligned}
& \liminf _{n \rightarrow \infty} \int_{0}^{\tau} \int_{\Omega} \frac{\partial W}{\partial F}\left(\nabla u^{n}\right): \nabla u^{n}-\overline{\frac{\partial W}{\partial F}(\nabla u)}: \nabla u^{n} \mathrm{~d} x \mathrm{~d} t \\
& \quad=\liminf _{n \rightarrow \infty} \int_{0}^{\tau} \int_{\Omega} \frac{\partial V}{\partial F}\left(\nabla u^{n}\right): \nabla u^{n}-\frac{\overline{\partial V}(\nabla u)}{\partial F}: \nabla u^{n} \mathrm{~d} x \mathrm{~d} t-2 \Lambda \int_{0}^{\tau} \omega(t) \mathrm{d} t
\end{aligned}
$$


where, by virtue of [4, Chapter 1, Theorem 5.1] and the property of weak lower semi-continuity of convex functionals,

$$
\begin{aligned}
\liminf _{n \rightarrow \infty} \int_{0}^{\tau} \int_{\Omega} \frac{\partial V}{\partial F}\left(\nabla u^{n}\right): \nabla u^{n}-\frac{\overline{\partial V}}{\partial F}(\nabla u): \nabla u^{n} \mathrm{~d} x \mathrm{~d} t \\
=\liminf _{n \rightarrow \infty} \int_{0}^{\tau} \int_{\Omega} V\left(\nabla u^{n}\right)+V^{*}\left(\frac{\partial V}{\partial F}\left(\nabla u^{n}\right)\right)-\frac{\overline{\partial V}}{\partial F}(\nabla u): \nabla u^{n} \mathrm{~d} x \mathrm{~d} t \\
\geq \liminf _{n \rightarrow \infty} \int_{0}^{\tau} \int_{\Omega} V^{*}\left(\frac{\partial V}{\partial F}\left(\nabla u^{n}\right)\right) \mathrm{d} x \mathrm{~d} t \\
\quad+\liminf _{n \rightarrow \infty} \int_{0}^{\tau} \int_{\Omega} V\left(\nabla u^{n}\right)-\frac{\partial V}{\partial F}(\nabla u): \nabla u^{n} \mathrm{~d} x \mathrm{~d} t \\
\geq \liminf _{n \rightarrow \infty} \int_{0}^{\tau} \int_{\Omega} V^{*}\left(\frac{\partial V}{\partial F}(\nabla u)\right)+V\left(\nabla u^{n}\right)-\frac{\partial V}{\partial F}(\nabla u): \nabla u^{n} \mathrm{~d} x \mathrm{~d} t \geq 0 .
\end{aligned}
$$

Combining (5.5)-(5.7) we arrive at the inequality

$$
\lambda \int_{0}^{\tau} k(t)(\omega(\tau-t)+\omega(t)) \mathrm{d} t \leq 2 \Lambda \int_{0}^{\tau} \omega(t) \mathrm{d} t \text { for any } \tau \in[0, T] .
$$

Now, our ultimate goal is to show that (5.8) implies $\omega \equiv 0$ on $[0, T]$ which amounts to (5.1). We proceed by induction assuming

$$
\omega \equiv 0 \text { on }\left[0, n \tau_{0}\right]
$$

where $\tau_{0}$ is chosen in such a way that

$$
k(t)>4 \frac{\Lambda}{\lambda} \text { on }\left(0, \tau_{0}\right) .
$$

Obviously, (5.9) holds for $n=1$. Assuming (5.9) for $n \geq 1$ we take $\tau=(n+1) \tau_{0}$ in (5.8) to obtain

$$
\int_{0}^{(n+1) \tau_{0}} k(t) \omega\left((n+1) \tau_{0}-t\right) \mathrm{d} t \leq 2 \frac{\Lambda}{\lambda} \int_{n \tau_{0}}^{(n+1) \tau_{0}} \omega(t) \mathrm{d} t
$$

where the left-hand side is estimated from below as

$\int_{0}^{(n+1) \tau_{0}} k(t) \omega\left((n+1) \tau_{0}-t\right) \mathrm{d} t \geq 4 \frac{\Lambda}{\lambda} \int_{0}^{\tau_{0}} \omega\left((n+1) \tau_{0}-t\right) \mathrm{d} t=4 \frac{\Lambda}{\lambda} \int_{n \tau_{0}}^{(n+1) \tau_{0}} \omega(t) \mathrm{d} t$ which yields the desired conclusion.

The relation (5.1) yields the energy inequality (1.17) and, together with (4.10), it implies (4.13) (cf. e.g. [4, Chapter 8, Corollary 1.3]).

Theorem 1.1 has been proved.

\section{REFERENCES}

[1] J.M. Ball, P.J. Holmes, R.D. James, R.L. Pego, and P.J. Swart. On the dynamics of fine structure. J. Nonlinear Sci., 1:17-70, 1991. MR 92d:35194

[2] P.G. Ciarlet. Mathematical elasticity: I. Three-dimensional elasticity. North-Holland, Amsterdam, 1988. MR 89e:73001

[3] P. Clément, G. Gripenberg, and S.-O. Londen. Schauder estimates for equations with fractional derivatives. Trans. Amer. Math. Soc., 352:2239-2260, 2000. MR 2000j:35161

[4] I. Ekeland and R. Temam. Convex analysis and variational problems. North-Holland, Amsterdam, 1976. MR 2000j:49001

[5] H. Engler. Global smooth solutions for a class of parabolic integrodifferential equations. Trans. Amer. Math. Soc., 348:267-290, 1996. MR 96k:35116 
[6] E. Fašangová and J. Prüss. Evolution equations with dissipation of memory type. In Topics in nonlinear analysis, J. Escher, G. Simonett, Editors, Birkhäuser, pages 211-250, 1999. MR 2000g:00092

[7] G. Friesecke and G. Dolzmann. Implicit time discretization and global existence for a quasilinear evolution equation with nonconvex energy. SIAM J. Math. Anal., 28:363-380, 1997. MR 97k:35170

[8] G. Friesecke and J.B. McLeod. Dynamics as a mechanism preventing the formation of finer and finer microstructure. Arch. Rational Mech. Anal., 133:199-247, 1996. MR 97h:35220

[9] H. Gajewski, K. Gröger, and K. Zacharias. Nichtlineare Operatorgleichungen und Operatordifferentialgleichungen. Akademie Verlag, Berlin, 1974. MR 58:30524a

[10] G. Gripenberg, Londen S.-O., and O. Staffans. Volterra integral and functional equations. Cambridge University Press, Cambridge, 1990. MR 91c:45003

[11] J.-L. Lions. Quelques méthodes de résolution des problèmes aux limites non linéaires. Dunod, Gautthier - Villars, Paris, 1969. MR 41:4326

[12] J.-L. Lions and E. Magenes. Problèmes aux limites non homogènes et applications, I. Dunod, Gautthier - Villars, Paris, 1968. MR 40:512

[13] R.L. Pego. Phase transitions in one-dimensional nonlinear viscoelasticity: Admissibility and stability. Arch. Rational Mech. Anal., 97:353-394, 1987. MR 87m:73037

[14] H. Petzeltová and J. Prüss. Global stability of a fractional partial differential equation. J. Int. Equations Appl., 12(3):323-347, 2000. CMP 2001:07

[15] J. Prüss. Evolutionary integral equations and applications. Birkhäuser, Basel, Boston, Berlin, 1993. MR 94h:45010

[16] P. Rybka. Dynamical modelling of phase transitions by means of viscoelasticity in many dimensions. Proc. Royal Soc. Edinburgh, 121 A:101-138, 1992. MR 93d:35164

[17] P. Rybka. The viscous damping prevents propagation of singularities in the system of viscoelasticity. Proc. Royal Soc. Edinburgh, 127 A:1067-1074, 1997. MR 98k:35191

[18] H. Triebel. Interpolation theory, function spaces, differential operators. VEB Deutscher Verlag der Wissenschaften, Berlin, 1978. MR 80i:46032a

Mathematical Institute of the Academy of Sciences of the Czech Republic, Žitná 25, 11567 Praha 1, Czech Republic

E-mail address: feireisl@math.cas.cz

Mathematical Institute of the Academy of Sciences of the Czech Republic, Žitná 25, 11567 Praha 1, Czech Republic

E-mail address: petzelt@math.cas.cz 